\title{
A better than perfect match
}

\author{
Entanglement, a mind-boggling form of correlations that exist between objects in the quantum world, is \\ helping to explain phenomena and jazzing up computing. But it looks as if much more may be in store.
}

\section{VlatkoVedral}

The concept of correlations is familiar to us from everyday experiences. We try to eat healthy food, drink less coffee and give up smoking because we believe that such behaviour is correlated to a higher life expectancy. We send our children to good schools in the belief that one day this will correlate with them having a better quality of life. Ultimately, as is the case for anyliving entity, it is our ability to recognize and adapt to these correlations that maximizes our chances of survival.

But why are events in nature correlated in the furst place? We don't really have an answer to this, although we are at least able to confirm the presence of correlations experimentally. Indeed, some of the most general and persistent correlations have been termed laws of nature', of which the second law of thermodynamics is a famous example. This states that things always flow from a more ordered to a less ordered state of affairs.

One would think we were fairly safe in assuming that correlations never exceed $100 \%$ (that is, that they cannot be better than perfect). If all children who go to good schools perform magnificently in life, we would say there is a $100 \%$ correlation between good schools and a successful life. You surely cannot get a better a correlation than this. But, odd as it may sound, correlations in nature can actually be better than perfect. This was first realized when physicists tried to infer the laws governing the behaviour of small objects; that is, in the study of quantum physics.

To qualify this paradox, imagine a simple two-state quantum system, such as the spin of an electron. Electrons are like small spinning-tops; each one rotates in its own way depending on the external circumstances. Just like a spinning-top, an electron can spin clockwise or counterclockwise in any given direction: horizontally, vertically, at $45^{\circ}$, and so on. Astonishingly, if we measure the electron spin at two different times, the correlations between these measurements can actually exceed any correlations allowed by classical physics.

Classically, spins at different times are expected to be correlated in the horizontal or vertical direction, so that if the first measurement of spin yields horizontal clockwise' so does the second. Real electrons, on the other hand, behave quantum mechanically; their spin measurements can be correlated in the vertical direction at the same time as in the horizontal direction (and all other directions!). This is because electrons can spin simultaneously in the dockwise and counterclockwise direction - something that no spinning-top can do.

As a consequence of this, two electrons can be more correlated in spin than anything allowed by classical physics. This is not just a neat mathematical trick but an effect that has been confirmed experimen-

There is a sense in which entangled quantum objects can be thought of as a single quasi-object. This way of looking at things is paramount to understanding many phenomena such as Bose condensation (the formation of very low-temperature fluids), superfluidity and superconductivity.

Recently, we have also realized that quantum entanglement allows us to process information more efficiently than can be done with conventional (classical) computers. Certain tasks, such as the factorization of large numbers, can be performed exponentially faster on a quantum computer than on any dassical computer. Whether such quantum correlations are alreadybeing used by living systems is not yet known, but there are some compelling indications that the answer might just again be 'yes.'

So, correlations are fundamental for our description and understanding of the world. In fact, it's tempting to say that things and events have no meaning in themselves, and that only the correlations between them are 'real.' This philosophy is known under the general name of relation-

tally many times. Such quantum correlations that exist between objects and events are known as 'entanglement.

A big question is whether entanglement is a wholly microscopic quirk, or whether it has any place in the large (macroscopic) world. Historically, this question has led to many apparent paradoxes in the quantum description of nature, but we now know that the answer is definitely 'yes.' For example, the magnetic behaviour of various solids is determined by the response of the electrons in the solid to the external magnetic field; the more correlated the spins of the electrons, the more they spin in the direction of the external field. A number of recorded measurements of magnetic responses of solids can only be explained if we assume that the electrons are entangled.

More surprisingly, this correlation between the spins of electrons and the direction of the external field happens in thermal equilibrium and at temperatures of up to $200 \mathrm{~K}$, making these observations very real indeed. Equilibrium implies that the system is not being externally driven into an entangled state but is only subject to environmental noise. Moreover, systems at higher temperatures experience more noise, which should destroy correlations. alism; Einstein's general theory of relativity is a shining example of its practical application in physics. Einstein was, however, unable to take relationalism one step further - to find a unified framework for general relativity and quantum physics. To this day this remains an open problem.

Could it be that this unification is proving elusive because quantum correlations (entanglement) have not been incorporated at the most fundamental level of its description? A view is now emerging according to which points in space and time can be thought of as correlated quantum objects, just like electrons in a typical solid. Can spatial and temporal distances between these points then be described as the amount of entanglement between them? Whatever the answer, one thing is certain: when it comes to entanglement, we have only just uncovered the tip of the iceberg.

Vlatko Vedral is professor of quantum information science at the University of Leeds, UK.

\section{FURTHER READING}

Tayloc, 1 C. Hidden Unity in Nature's Laws (Cambridge Univ. Press, 2001).

Born, M. Natural Philosophy of Couse and Chance (Dover, 1964).

Brooks, M.New Sci. 181, 32(2004).

(

